\title{
Quality model for multi-level course specification and delivery
}

\author{
Brian Garner \\ School of Information Technology, Deakin University.
}

\begin{abstract}
The productivity imperative imposed during the 90's resulted in active consideration and development of multi-level courses within universities. The move to electronic delivery of such courses, facilitated at Deakin University since 1996 by the use of learning management tools exacerbated concerns regarding the quality of education available to both undergraduates, and more recently, to full fee postgraduate students. Successful models for multi level course specification and delivery have not been widely canvassed in the academic community. Pilot projects in the School of Information Technology during the 90's included courses in Electronic Data Interchange (EDI) and Computer Audit (CA) supported by electronic mail. This paper addresses the critical success factors identified during the past decade and describes a proposed model for successful specification and evaluation of multi-level course proposals.
\end{abstract}

Key words: Quality Model; Multi Level Course Specification; Learning Support Dynamics; Learning Intelligence; Causal Models

\section{INTRODUCTION}

Distance education providers have not actively canvassed successful models for multi-level course specification and delivery in the academic community despite their widespread adoption. Academics involved in the electronic design and delivery of course materials (E-Learning) at multiple levels, particularly postgraduate courses, are consequently at risk, given the paucity of relevant educational models appropriate to the experiential learning requirements.

The original version of this chapter was revised: The copyright line was incorrect. This has been corrected. The Erratum to this chapter is available at DOI: 10.1007/978-0-387-35700-3_33 
Garner and Lawrence (1998) proposed a model based on knowledge domains for scoping educational and support issues for electronic commerce. This model is applicable to asynchronous learning frameworks, subject to the adoption of relevant meta-knowledge acquisition strategies, but did not support the requisite management processes for quality assessment. However, the limited capacity of generic, online platforms currently available to support a dynamic learning environment for mature students requiring the integration of didactic knowledge with experiential knowledge and corresponding reference models is no less a concern! More recently, the risk of learning dissonance using Web-based platforms has been highlighted by McKay, Garner and Okamoto (2002).

Studies of support requirements for experiential learning at Deakin University have identified the importance of learning support dynamics in effective interaction with mature-age students. The results of this review have been presented elsewhere, (Garner, 2002), and the need for a learning intelligence environment adumbrated. The additional complexity imposed by multi-level course specification and delivery is currently being addressed through the introduction of causal models within the respective knowledge domain for assessing the quality of student interaction.

\section{KNOWLEDGE DOMAINS}

The principal domains justifying the development of a methodology for multi-level course specification and delivery in the nineties arose from the recognition of full-fee postgraduate course opportunities, while simultaneously, the opportunity to enrich undergraduate electives in these areas was enthusiastically embraced. In effect, the harsh funding climate precluded undergraduate electives, such as Computer Audit, that were not otherwise underpinned by full-fee postgraduate offerings! This paper draws principally on Deakin's experience with two programs:

Electronic Data Interchange (EDI): Graduate Diploma launched in 1991.

Computer Audit: Masters and third-year undergraduate level units.

The characteristics of these domains may be summarised as:

- Wide-ranging application in business and Government.

- Ontological complexity for new practitioners (eg Forensic audit).

- Extensive professional practice underpinning their application.

- Historical evolution of the respective concepts and craft knowledge.

- Published Global Standards and Reference Models for Application level messages and risk management. 
- Integration of didactic knowledge required from several technical areas (eg: data communications, data modelling, software engineering)

- Inclusion of experiential knowledge supplied by external Experts and Workshop exposure to tacit knowledge of practitioners.

\section{CRITICAL SUCCESS FACTORS}

The postgraduate students undertaking studies in these areas were unusually diverse in their backgrounds, prior knowledge level and motivation. The methodology and Quality Model adopted were, therefore, crucial. Critical Success Factors (CSFs) were identified in securing quality endorsement by the students, initially based on questionnaire responses. The Graduate Diploma of EDI was also endorsed by an EDI Committee of the All India Management Association (AIMA), based in Delhi. AIMA subsequently delivered this program as their Professional Diploma across India, supported by EDI Workshops conducted in India by Deakin University.

The Critical Success Factors identified over the past decade may be summarised as:

- Blending of theory and practice, supported by Campus-based Workshops. It is impractical, for example, to motivate students to learn methodologies, including research methodology, for example, without experiential learning support.

- Assignments should identify the experiential knowledge requirements, and in many cases, syndicated project work (ie collaborative projects) is essential to ensure that student diversity is maintained by empowering students to compensate for their lack of prior knowledge in certain areas.

- Case studies and exemplars should be drawn from business and government, rather than from textbooks, to ensure that appropriate solution contexts can be identified by students and the respective knowledge sources (formal and/or tacit knowledge sources) consulted!

- Evaluation paradigm supported by published criteria/standards! The quality of the evaluation paradigm is paramount for ALL students, albeit for differing reasons! For example, justification of the assignment evaluation process in the context of stated learning objectives becomes more difficult, when selection of the correct knowledge level is a prime factor in the quality of the result achieved by the student/team.

- Provision of a knowledge acquisition process for students is essential to assist them in their interpretation of published guidelines on the use of standards or reference models in a given contextual framework. 
- Effective integration of study material from diverse sources with the requisite pedagogical knowledge, including reference sources/ontologies, has limited the contribution of practitioners, other than through workshop presentations .

- External review of the Unit specifications and proposed content/delivery mode, in the light of the foregoing CSFs, is typically difficult and beyond the capabilities of junior academics lacking the requisite experiential knowledge (industrial practice).

- Most recently, the role of machine intelligence and the need for causal models to provide hypothesis generation capabilities and explanations at multiple abstraction levels has been explored.

\section{LEARNING SUPPORT DYNAMICS}

Recognition of the overarching role of learning support dynamics in any quality endorsement process arose from research at Deakin University in a number of key areas:

- A longitudinal study in 1997-98 of the effectiveness of a telematics helpdesk for external students of computing identified a wide disparity between students' responses and tutors' beliefs (Coldwell, Coldwell \& Garner, 1999). The conclusion was drawn that an intelligent helpdesk, based on online knowledge support, was the most promising model for improving learning effectiveness by offering a thematic interpretation of student requests!

- A common mechanism for providing feedback to students, whether the course is based on a textbook or uses original material, is to provide student exercises and sample answers which for undergraduate courses may be drawn from textbooks. In multi-level courses with integrated experiential knowledge and/or external reference models, this mechanism would typically be deficient, due to the multiple levels of abstraction/explanation that are necessary to satisfy all students!

- Knowledge engineering paradigms at Deakin University, particularly the use of goal interpretation as a knowledge acquisition mechanism (Lukose, 1992), have potentially offered new solutions for automated student support. Heuristic abstraction for automated case management, for example, is deemed to provide a core technology for learning intelligence environments (Garner, 2002).

- Causal chains have provided a useful mechanism in Expert systems and $\mathrm{CBR}$ for generating explanations from the inferencing process. 
However, as commonly implemented, such mechanisms are restricted to rule formats.

- The advent of other more general techniques, such as data mining, have not been exploited in providing online, learning support dynamics to the author's knowledge.

\section{CORE COMPONENTS FOR SOLUTIONS ARCHITECTS IN ENABLING LEARNING INTELLIGENCE}

The role of solutions architects in learning intelligence was adumbrated in an earlier paper (Garner, 2002), focussing on conceptual ontology research (Raban \& Garner, 2001) and the overarching need for a knowledge dictionary personalised to individual requirements.

Current research by the author into learning intelligence environments presumes an architecture incorporating three layers:

Level 1: Learning management software tool (eg FirstClass, WebCT)

Level 2: Learning (Courseware) Objects/Formal Knowledge (Reference models)

Level 3: Personalised Knowledge Dictionary/Human Computer Interface and Context mining/mediation process, supported by an intelligent helpdesk for tacit knowledge elicitation.

The core components in prospect for level 3 may be summarised as follows:

(a) Automated Case Management Toolkit:

Context mining and knowledge mediation (Philp \& Garner, 2001) is being implemented using Automated case management, in which heuristic abstraction has been used (offline) to determine script-based, intelligent case managers that implement appropriate context selection/mediation for bridging the gap between the students' knowledge level and the task knowledge requirements.

(b) Personalised Knowledge Dictionary

The Knowledge Dictionary architecture developed by Garner \& Lukose (1991) as an educational tool for training line operators within Pilkingtons Australia, is shown in Figure 1. It is being adapted for customisation within the specified learning intelligence requirement to store:

Ontology mappings for alias recognition; Knowledge levels achieved (Explanation based); Conditions-of-the-learner (Assertion testing); Cognitive style (Event likelihood)

(c) Hypothesis Generation Paradigm: 
In the process of enabling machine intelligence, a paradigm for automated hypothesis generation was developed, based on earlier research at Deakin University. The reader should consult the doctoral thesis by Fan Chen (1996) for further details of the basic paradigm.

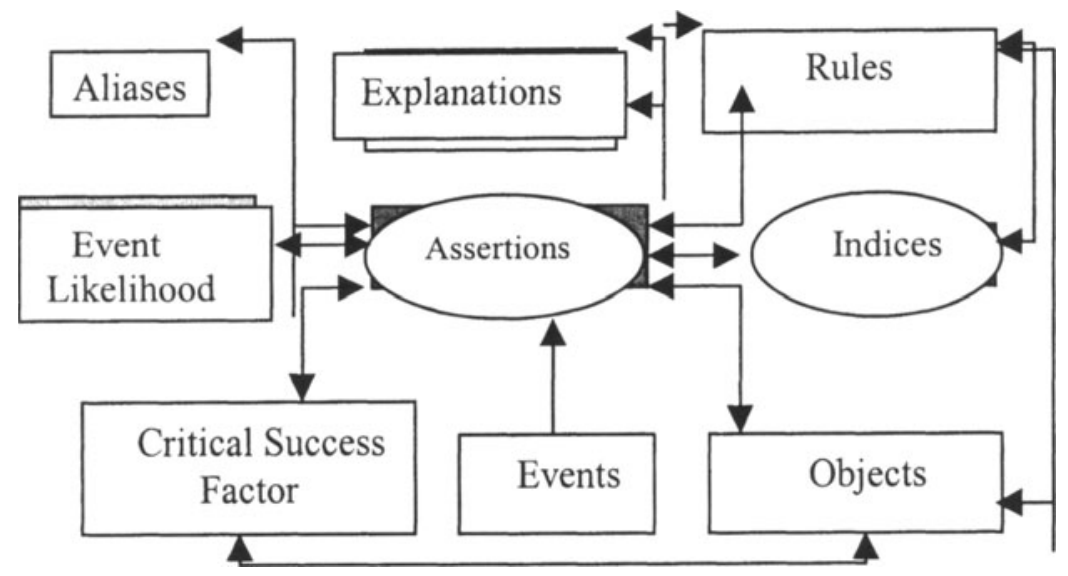

Figure 1. Knowledge Dictionary Structure

\section{(d) Causal Models:}

The scope for artificial intelligence in automated learning support is quite promising for multi-level instruction in a given knowledge domain. The current phase of this research is focussed on pre-defined causal models, which are themselves generated from the specified learning processes and from the requisite abstractions (meta-knowledge). Causal models provide, in effect, a set of relationships between the diverse knowledge/reference sources within a specified learning process and the possible contexts that might exist during goal interpretation by the student. This mechanism ensures that experiential knowledge is correctly blended with the dynamics of the discovery process, while providing a novel implementation of explanation-based learning ( $\mathrm{cf}$ Schank) at the appropriate level.

Causal models may also be implemented as an enabling technology, supported by appropriate reasoning themes, with immediate application to important quality issues in learning effectiveness: eg.

- Monitoring the conditions-of-the-learner, other than by progressive (repeat) testing, to establish prior knowledge requirements!

- Study of the effectiveness of learning intervention using context \& event mining(episodic contexts) to evaluate the effect of context changes on students progress with individual or group exercises.

- Understanding the ontological support requirements for task analysis in problem solving environments. 


\section{SUMMARY OF PROPOSED QUALITY MODEL}

The essence of the proposal given in Figure 2 lies in the use of causal models to provide a dynamic learning environment, based on the integration of several core components for context-mediation, tailored to the knowledge level specified for student assessment., given the experiential knowledge requirements of the domain and pre-defined course objectives. The design and implementation of causal models follows logically from prior work at Deakin (and elsewhere) over the past two decades in knowledge engineering, using conceptual graphs as the knowledge representation scheme and the associated graph-based inference engine. (Tsui, Garner \& Lukose, 1997). Quality outcomes are assured through the automation of context-mediation, through monitoring of the effectiveness of learning intervention and through online assessment of the conditions-of-the-learner.

\section{REFERENCES}

Chen, Fan, (1996) : Hypothesis Generation for Management Intelligence; Ph.D. Thesis, Faculty of Science \& Technology, School of Computing \& Mathematics, Deakin University, Victoria 3217, Australia

Coldwell, J., Coldwell, R. and Garner, B.J. (1999). Computer-based Helpdesks: A Major facility to aid Course Authoring and Delivery to external students. Report to Telematics Trust (Melbourne).

Garner, B.J. (2002): Role of Solutions Architects in Learning Intelligence. Invited paper in eLearning Conference on Design and Development: International Best Practice to Enhance Corporate Performance - Edited by Dr. E. McKay; October 21-25, Melbourne, Australia, RMIT Informit Library. 18-25

Garner, B.J and Lawrence, E. (1998). Knowledge Domains in Scoping Educational and Support Issues for Internet Commerce in 'Proceedings of Eleventh International BLED Electronic Commerce Conference', pp 109-117; BLED Slovenia

Garner, B.J., and Lukose, D (1991) : Expert Advisor utilising Goal Interpretation Methods; Proceedings of the First World Congress on Expert Systems, Florida, USA.

Lukose, D. (1992). Goal interpretation as a knowledge acquisition mechanism, Ph.D.Thesis. Faculty of Science and Technology, School of Computing and Mathematics. Deakin: Victoria, Australia.

McKay, E., Garner, B.J and Okamoto, T (2002). Understanding the Ontological Requirements for Collaborative Web-based Experiential Learning; International Conference on Computers in Education 2002, Auckland NZ, IEEE Computer Society, 356-357

Philp, B.A and Garner, B.J (2001): Knowledge Mediation in Software Quality Engineering; ASWEC 2001, Canberra, August 26-28

Raban, R and Garner, B.J. (2001, September 19-21) Ontological Engineering for Conceptual Modelling, K1-2001, Vienna.

Tsui, E., Garner, B.J., and Lukose, D (1997); EGP: Extendible Graph Processor. Published in 'Conceptual Structures: Fulfilling Peirce's Dream', Lecture Notes in Artificial Intelligence 1257, pp 594-597. 


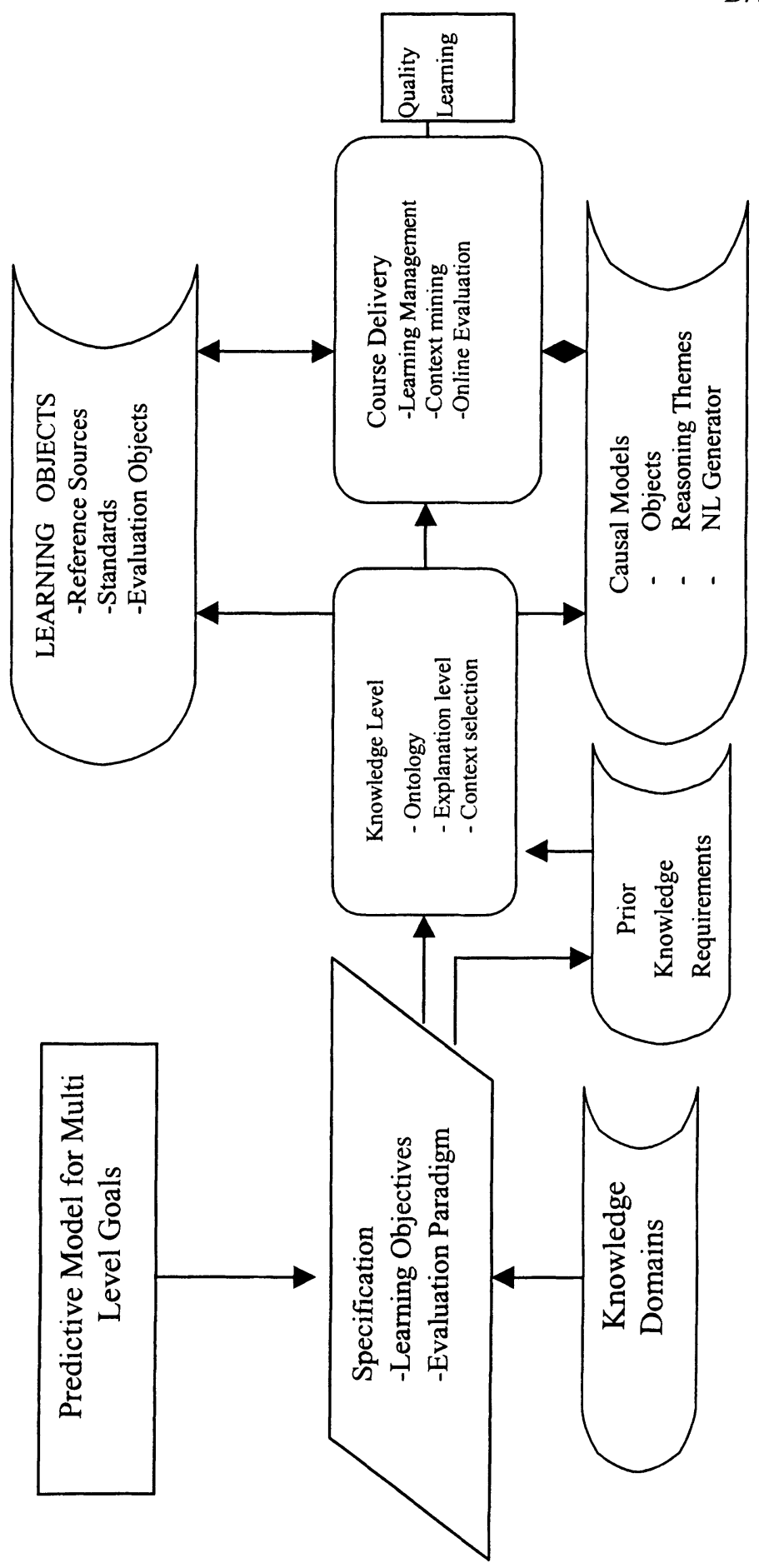

Figure 2 Proposed Quality Model for Multi-Level Course Specification and Delivery 